\title{
Epidemiology, natural history, and optimal management of neurohypophyseal germ cell tumors
}

\author{
Hirokazu Takami, MD, PhD, ${ }^{1}$ Christopher S. Graffeo, MD, MS, ${ }^{1}$ Avital Perry, MD, ${ }^{1}$ \\ Caterina Giannini, MD, PhD, ${ }^{2}$ and David J. Daniels, MD, PhD' \\ Departments of ${ }^{1}$ Neurologic Surgery and ${ }^{2}$ Laboratory Medicine and Pathology, Mayo Clinic, Rochester, Minnesota
}

\begin{abstract}
OBJECTIVE Intracranial germ cell tumors (iGCTs) often arise at the neurohypophysis, their second most common origination, following the pineal region. Neurohypophyseal iGCTs present with stereotypical symptoms, including pituitary dysfunction and visual field deficit, due to their suprasellar location. The goal of this study was to present a large, longitudinal single-institution experience with neurohypophyseal iGCTs to better understand their natural history and identify opportunities for further improvement in treatment outcomes.
\end{abstract}

METHODS This is a retrospective, single-institution cohort study of neurohypophyseal iGCTs treated between 1988 and 2017, with a focus on the epidemiology, presentation, natural history, and treatment.

RESULTS Thirty-five neurosurgically managed patients met inclusion criteria; the median age was 18 years ( 3 months to 49 years), and $74 \%$ of patients were male $(n=26)$. Thirty-one tumors were germinomas, and 4 were nongerminomatous iGCTs. Presenting symptoms included pituitary insufficiency in $76 \%(n=25)$, visual deficit in $45 \%(n=15)$, and diabetes insipidus (DI) in 61\% $(n=20)$ of patients. Index symptoms included isolated DI in $10(36 \%)$, isolated hormone deficiency in $14(50 \%)$, and concomitant Dl and hormone deficiency symptoms in $4(14 \%)$. Radiographic diagnostic latency was common, occurring at a median of 363 days (range 9-2626 days) after onset of the first symptoms and was significantly associated with both DI and hormone deficiency as the index symptoms (no DI vs DI: 360 vs 1083 days, $p=0.009$; no hormone deficiency vs hormone deficiency: 245 vs 953 days, $p=0.004$ ). Biochemical abnormalities were heterogeneous; each pituitary axis was dysfunctional in at least 1 patient, with most patients demonstrating at least 2 abnormalities, and pretreatment dysfunction demonstrating a nonsignificant trend toward association with long-term posttreatment hormone supplementation. Among germinomas, whole-brain or whole-ventricle radiotherapy demonstrated significantly improved progression-free and overall survival compared with local therapy ( $p=0.009$ and $p=0.004$, respectively).

CONCLUSIONS Neurohypophyseal iGCTs are insidious tumors that may pose a diagnostic dilemma, as evidenced by the prolonged latency before radiographic confirmation. Serial imaging and close endocrine follow-up are recommended in patients with a characteristic clinical syndrome and negative imaging, due to the propensity for radiographic latency. Pretreatment biochemical abnormalities may indicate higher risk of posttreatment pituitary insufficiency, and all patients should receive careful endocrine follow-up. Local radiotherapy is prone to treatment failure, while whole-ventricle treatment is associated with improved survival in germinomas.

https://thejns.org/doi/abs/10.3171/2019.10.JNS191136

KEYWORDS germ cell tumor; neurohypophysis; hormone deficiency; diabetes insipidus; prognosis; oncology

I NTRACRANIAL germ cell tumors (iGCTs) are rare CNS neoplasms that predominantly occur in pediatric and young adult patients. According to the Central Brain Tumor Registry of the United States, the incidence in the US was $3.8 \%$ among patients younger than 20 years. ${ }^{21}$ Midline tumors, iGCTs most frequently arise in the pineal region, followed by the neurohypophysis, which is defined as the pituitary gland proper, its infundibulum, and the hypothalamus. The WHO classification of CNS tumors defines 5 histopathological iGCT subtypes: germinoma, mature and immature teratoma, yolk sac tumor, choriocarcinoma, and embryonal carcinoma. ${ }^{15}$ However, in practice,

ABBREVIATIONS ACTH = adrenocorticotropic hormone; $\mathrm{ADH}=$ antidiuretic hormone; $\mathrm{COG}=\mathrm{Children's} \mathrm{Oncology} \mathrm{Group;} \mathrm{CSI}=\mathrm{craniospinal}$ irradiation; $\mathrm{DI}=$ diabetes insipidus; FSH = follicle-stimulating hormone; GH = growth hormone; iGCT = intracranial germ cell tumor; IGF = insulin-like growth factor; LH = luteinizing hormone; NGGCT = nongerminomatous germ cell tumor; OS = overall survival; PGC = primordial germ cell; $P F S=$ progression-free survival; TSH = thyroid-stimulating hormone; WBI = wholebrain irradiation; $\mathrm{WVI}=$ whole-ventricle irradiation.

SUBMITTED April 22, 2019. ACCEPTED October 14, 2019.

INCLUDE WHEN CITING Published online February 7, 2020; DOI: 10.3171/2019.10.JNS191136. 
tumors commonly demonstrate mixed features and have characteristics of multiple histopathological subtypes. Correspondingly, the more clinically meaningful iGCT division is between germinomas and nongerminomatous germ cell tumors (NGGCTs).

Despite this heterogeneous nature of GCTs, primordial germ cells (PGCs) have been suggested as the common cell of origin. ${ }^{19,28}$ It is now hypothesized that totipotent PGCs during the migrating stage from the yolk sac to the genital ridge gain copy number alteration and/or mutational insults, which leads to mismigration of PGCs to the CNS and cause tumorigenesis. ${ }^{8,20,26}$ Although approximately $60 \%$ of iGCTs were shown to harbor a mutation in either the MAPK or PI3K pathway, an alternative pathogenic molecular background remains to be clarified.12

Neurohypophyseal iGCTs manifest stereotypical symptoms due to their anatomical location, including pituitary insufficiency, visual deficits attributable to optic pathway compression or invasion, and hydrocephalus in large tumors compressing the third ventricle. ${ }^{16}$ Standardof-care treatment involves protocol-based chemotherapy and radiation therapy, with the exception of mature teratomas, which do not respond to chemo- and/or radiation therapy and therefore require resection. According to the Children's Oncology Group (COG) study protocol, localized germinomas and NGGCTs are treated with platinum-based chemotherapy and whole-ventricle irradiation (WVI)/focal irradiation. In Europe, according to the SIOP-CNS-GCT-96 trial (clinicaltrials.gov), germinoma is treated with either low-dose craniospinal irradiation (CSI) or chemotherapy, while NGGCT is treated with chemotherapy followed by focal irradiation or CSI, depending on the presence of metastases. ${ }^{5,6}$ As a common practice, iGCTs are diagnosed based on clinical presentation and tumor markers along with imaging findings if a case presents with typical findings, although there are controversies regarding the necessity of histopathological diagnosis and the best cutoff values for tumor markers. ${ }^{18}$

The neurohypophysis is a common site for iGCTs, second only to the pineal region, with bifocal neurohypophyseal/pineal lesions regularly reported. Although the majority of bifocal tumors are diagnosed as germinomas, rare cases have demonstrated bifocal yolk sac tumors and even bifocal primitive neuroectodermal tumors, reaffirming the need for cautious follow-up, as well as the potential benefits of surgical biopsy for definitive histopathological diagnosis. ${ }^{2,22}$ Germinoma potentially disseminates along the CSF space, which is clearly visualized with the aid of neuroendocopy; ${ }^{23}$ generally speaking, bifocal germinoma cases are safely treated with WVI with chemotherapy, although some controversy persists regarding possible indications for CSI in this patient population. ${ }^{30}$

Germinomas are the dominant neurohypophyseal histological entity and demonstrate an excellent clinical response to chemo- and radiation therapy, which achieves a good prognosis in approximately $90 \%$ of cases. ${ }^{1,6,16}$ Correspondingly, management of both disease- and treatmentrelated symptoms - in particular, hormone deficiency syndromes-is critical to the long-term care of these young patients. In parallel, early detection and diagnosis have been shown to minimize morbidity and mortality; there- fore, the goal of our study was to present a large, longitudinal single-institution experience with neurohypophyseal iGCTs to better understand the natural history and identify opportunities for further improvement in treatment outcomes..$^{14}$

\section{Methods}

A comprehensive, prospectively maintained neurosurgical patient registry was retrospectively queried for patients presenting with primary CNS iGCTs between 1988 and 2017. Captured clinical endpoints included age, sex, presenting symptoms, lesion location and size, histopathological diagnoses, surgical details, chemotherapy and/or radiation treatment details, recurrence, and vital status at last follow-up. All final histopathological diagnoses were made or confirmed at our institution. All pertinent components of the study were approved by our institutional review board. Statistical analysis included the Wilcoxon test for nonparametric values, log-rank test for survival data, and McNemar test for paired categorical data; multivariate modeling was completed via Cox regression analysis. All statistical analysis was carried out using JMP 13 (SAS Institute Inc.). An alpha level of 0.05 was used to define statistical significance.

\section{Results \\ Demographics and Pathologic Diagnoses}

At our institution, 98 iGCT patients were treated during the study period, including 35 with neurohypophyseal tumors. Of 98 cases, 80 were primary and 18 were metastases from other organs, including testis and ovary. The 80 primary cases included 47 germinomas, 5 mature teratomas, 4 immature teratomas, 1 yolk sac tumor, 1 choriocarcinoma, 10 cases of mixed germinoma and NGGCT, 1 case of mixed NGGCT, and 11 iGCTs not otherwise specified. Among the 35 neurohypophyseal tumors, histopathological diagnosis was made in 32 cases, and 3 cases were diagnosed based on clinical findings, including imaging appearance, clinical manifestations, and tumor markers. All included tumors were primary (not metastatic) CNS disease; multifocal lesions were included, provided that at least 1 tumor occurred in the neurohypophysis $(n=15$ concomitant neurohypophyseal and pineal lesions; $\mathrm{n}=3$ polycentric lesions).

Age distribution data were stratified by 5 -year increments, with the highest disease incidence noted among patients aged 15-19 years, and an overall median age of 18 years ( 3 months to 49 years) and mean 18.4 years (Fig. 1). The female-to-male sex distribution was approximately 1:3 (9:26). Thirty-one (89\%) of the iGCTs were germinomas; the remaining 4 cases included 1 mature teratoma, 1 immature teratoma, and 2 mixed iGCTs (germinoma + immature teratoma + yolk sac tumor + embryonal carcinoma; and germinoma with syncytiotrophoblastic giant cells + mature teratoma). Bifocal lesions were histopathologically confirmed as germinoma in all locations.

\section{Tumor Markers and Treatment}

In all but 3 cases, MRI studies were available for re- 

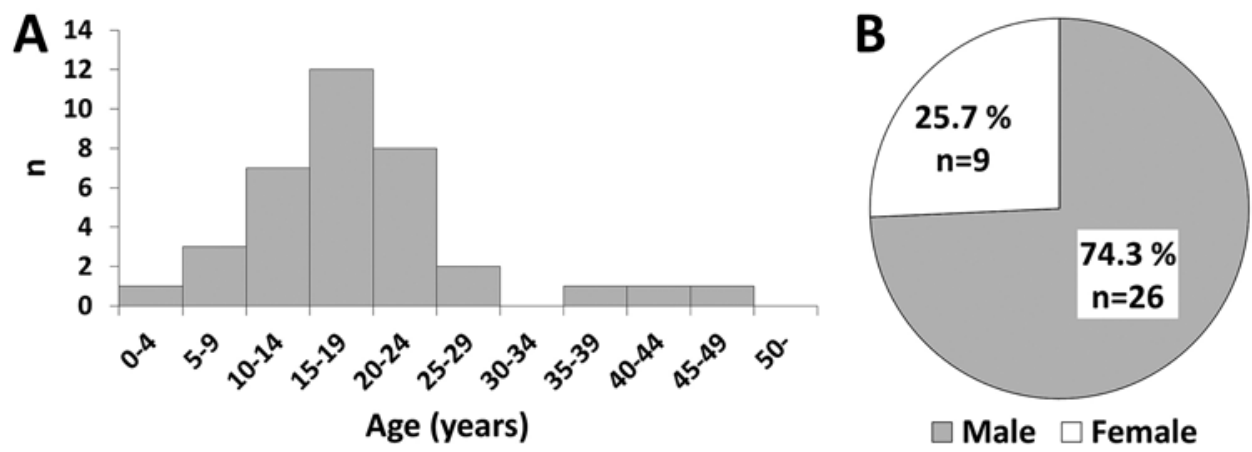

FIG. 1. Age- (A) and sex- (B) based distributions of 35 patients with neurohypophyseal iGCTs. The median and mean ages were 18 years.

view. The full neuraxis was imaged in 24 cases to rule out dissemination; 2 of these cases were positive, both of which were treated with CSI. CSF cytology was tested in 23 cases, among which 4 were positive. Pretreatment tumor markers were documented in 25 cases; among 23 cases of germinoma with available data, 2 cases demonstrated human chorionic gonadotropin levels elevated beyond the standard criteria of NGGCTs (serum or CSF total human chorionic gonadotropin > $50 \mathrm{IU} / \mathrm{L}$ ). Among the 32 cases diagnosed based on histopathological findings, specimens were obtained through endoscopic surgery ( $\mathrm{n}$ $=9)$, stereotactic needle biopsy $(\mathrm{n}=5)$, open craniotomy procedure $(n=15)$, and unknown procedures $(n=3)$.

After histopathological or clinical diagnosis, all patients underwent adjuvant treatment including chemotherapy and radiation therapy, except for 1 patient with a mature teratoma. Treatment regimens were based on updated COG and/or institutional protocols, depending on the time of treatment. One germinoma in the medulla was treated as an NGGCT with 6 cycles of carboplatin/etoposide alternating with ifosfamide/etoposide followed by CSI and focal irradiation based on the ACNS0122 study (clinicaltrials.gov). ${ }^{9}$ Most of the other germinoma cases were treated based on the ACNS0232 study (radiation alone or chemotherapy followed by radiation), ACNS1123 (chemotherapy followed by WVI + focal irradiation), or the institutional protocol. The protocols followed were chemotherapy and focal irradiation for 10 cases, focal irradiation only for 2 cases, chemotherapy and CSI for 12 cases, CSI only for 3 cases, chemotherapy and WVI for 2 cases and WVI only for 1 case. The mature teratoma was resected without adjuvant therapy. Three other NGGCT cases were treated with chemotherapy, followed by focal irradiation.

Three of 31 germinomas required second-look surgery, which yielded mature teratoma $(\mathrm{n}=2)$ or fibrous tissue $(\mathrm{n}$ $=1$ ) as the histopathological diagnoses, but no further attempt to resect the tumor was made if the diagnosis was germinoma during the initial workup and treatment.

\section{Complications}

Among 32 surgical cases, there was 1 wound infection with abscess formation following craniotomy. Rare complications included anosmia and diplopia $(\mathrm{n}=1)$, Parinaud syndrome after second-look surgeries for neurohypophyseal mature teratoma/pineal fibrous tissue $(n=1)$, third and sixth nerve palsies after subtotal resection of a neurohypophyseal germinoma $(\mathrm{n}=1)$, homonymous hemianopia after gross-total resection of a neurohypophyseal $\operatorname{NGGCT}(n=1)$, and new seizures $(n=3)$.

\section{Comparison With Pineal GCTs}

The characteristics of neurohypophyseal iGCTs were compared with those of pineal iGCTs. There were 20 neurohypophyseal, 40 pineal, and 15 bifocal cases. The neurohypophyseal cohort was more sex balanced $(\mathrm{p}=0.0047)$, had more germinomas than NGGCTs $(p<0.0001)$, and presented with more endocrine symptoms (pituitary insufficiency in general and diabetes insipidus [DI], both $\mathrm{p}<$ 0.0001 ) than pineal-only cases (Table 1 ).

\section{Radiographic Latency as a Function of Index and Presenting Symptoms}

Due to the complex, often halting course iGCT patients experience between first symptoms and definitive diagnosis, we distinguished between the index symptoms, defined as the initial disease features reported in the patient history, and presenting symptoms, defined as all disease features documented at the time of their first medical assessment. Presenting symptoms were reported in 33 cases, which included 15 instances of visual deficit (45\%), 25 hormone deficiency syndromes (76\%), and 20 cases of DI (61\%; Fig. 2A).

Index symptoms were clearly documented in 28 patients and included isolated DI in 10 (36\%), isolated hormone deficiency in $14(50 \%)$, and concomitant DI and hormone deficiency symptoms in 4 (14\%) patients. Among the 14 patients with DI as an index symptom, 7 underwent MRI within 1 year; 5 of these studies were negative. Subsequent neuroimaging ultimately diagnosed neurohypophyseal tumors in all individuals; however, the latency periods were $150,315,889,942$, and 1366 days. In this last, most prolonged radiographic delay, MRI studies were persistently negative at 918 days, subsequent to which the first MRI study with positive findings was conducted 1366 days after initial symptomatic presentation (Fig. 2B). Compared with patients without DI as an index symptom, the latency period from symptomatic onset to diagnosis was significantly longer among patients who developed early DI (1083 vs 360 days, $p=0.009$; Fig. 2C). Similarly, patients whose 
TABLE 1. Comparison of clinical manifestations based on tumor location

\begin{tabular}{|c|c|c|c|c|}
\hline & Neurohypophysis & Pineal Gland & Both & $p$ Value $^{*}$ \\
\hline No. of patients & 20 & 40 & 15 & \\
\hline F/M ratio & $6: 14$ & $1: 39$ & $3: 12$ & 0.0047 \\
\hline Female sex (\%) & 30.0 & 2.5 & 20.0 & \\
\hline Age, yrs & & & & 0.22 \\
\hline Range & $0-49$ & $7-46$ & $9-42$ & \\
\hline Median & 19 & 15.5 & 17 & \\
\hline Mean & 18.5 & 16.7 & 18.4 & \\
\hline Histology† & & & & $<0.0001$ \\
\hline Germinoma & 16 & 15 & 15 & \\
\hline Mature teratoma & 1 & 2 & 0 & \\
\hline Immature teratoma & 1 & 2 & 0 & \\
\hline MaligT & 0 & 0 & 0 & \\
\hline Yolk sac tumor & 0 & 1 & 0 & \\
\hline Embryonal carcinoma & 0 & 0 & 0 & \\
\hline Choriocarcinoma & 0 & 1 & 0 & \\
\hline Mixed iGCT & 2 & 8 & 0 & \\
\hline Mixed NGGCT & 0 & 0 & 0 & \\
\hline GCT not otherwise specified & 0 & 11 & 0 & \\
\hline \multicolumn{5}{|l|}{ Presenting symptoms } \\
\hline Pituitary insufficiency & $73.7 \%(14 / 19)$ & $8.8 \%(3 / 34)$ & $78.6 \%(11 / 14)$ & $<0.0001$ \\
\hline DI & $52.6 \%(10 / 19)$ & $0.0 \%(0 / 31)$ & $71.4 \%(10 / 14)$ & $<0.0001$ \\
\hline Visual deficit & $47.4 \%(9 / 19)$ & $28.6 \%(10 / 35)$ & $42.9 \%(6 / 14)$ & 0.21 \\
\hline
\end{tabular}

index symptoms included a hormone deficiency syndrome had significantly increased latency periods prior to diagnosis (953 vs 245 days, $p=0.004$; Fig. 2D).

\section{Pituitary Insufficiency: Biochemical and Clinical Profiles}

Data on laboratory assessments at presentation were incomplete; however, as available, we captured parameters pertinent to the major pituitary axes, including adrenocorticotropic hormone (ACTH; corticotrophin and cortisol), thyroid-stimulating hormone (TSH; TSH and free T4), growth hormone $(\mathrm{GH})$ axis $(\mathrm{GH}$, insulin-like growth factor [IGF]-1, IGF-BP3), testosterone, luteinizing hormone/ follicle-stimulating hormone ( $\mathrm{LH} / \mathrm{FSH})$, and prolactin. Quantitative data were dichotomized as normal and abnormal using institutionally validated reference ranges with age- and sex-adjusted thresholds. In patients with at least 2 years of follow-up, long-term hormone supplementation was also tracked.

Biochemically confirmed pretreatment pituitary function abnormalities included ACTH deficiency in 11 of 20 (55\%), TSH deficiency in 7 of 19 (37\%), GH/IGF-1 in 11 of $17(65 \%)$, testosterone in 13 of 14 (93\%), LH/FSH deficiency in 5 of $12(42 \%)$, and hyperprolactinemia in 16 of 24 (67\%; Fig. 3A). At last follow-up, chronic hormone supplementation requirements included corticosteroids in 24 of 32 (75\%), thyroid hormone in 22 of 32 (69\%), GH in
5 of $29(17 \%)$, testosterone in 17 of $29(59 \%)$, and antidiuretic hormone (ADH) in 23 of 32 (72\%; Fig. 3B) cases.

Dichotomized, paired data for preoperative biochemical status and postoperative hormone supplementation were tested for correlation. No significant associations were observed; however, a large proportion of patients with preoperative hormone deficiency syndromes were noted to require supplementation in follow-up, including corticosteroids in $100 \%$, TSH in $100 \%$, GH in $38 \%$, testosterone in $73 \%$, and $\mathrm{ADH}$ in $94 \%(\mathrm{p}=0.13, \mathrm{p}=0.07, \mathrm{p}=$ $0.22, p=0.25$, and $p=0.22$, respectively; Fig. $3 \mathrm{C}$ ).

\section{Prognosis and Radiation Coverage}

Radiation field size was tested in association with prognosis in germinomas $(n=31)$. Progression-free survival (PFS) and overall survival (OS) were both significantly longer after whole-brain irradiation (WBI) or WVI, compared with local treatment $(p=0.009$ and $p=0.004$, respectively). Several patients with unifocal disease did not undergo platinum-based chemotherapy. This prompted a multivariate analysis to control for the potential confounder, which confirmed that both WBI and WVI were independently associated with statistically significant improvements in PFS and OS, compared with local radiation $(p=0.009$ and $p=0.025$, respectively; Fig. 4). 

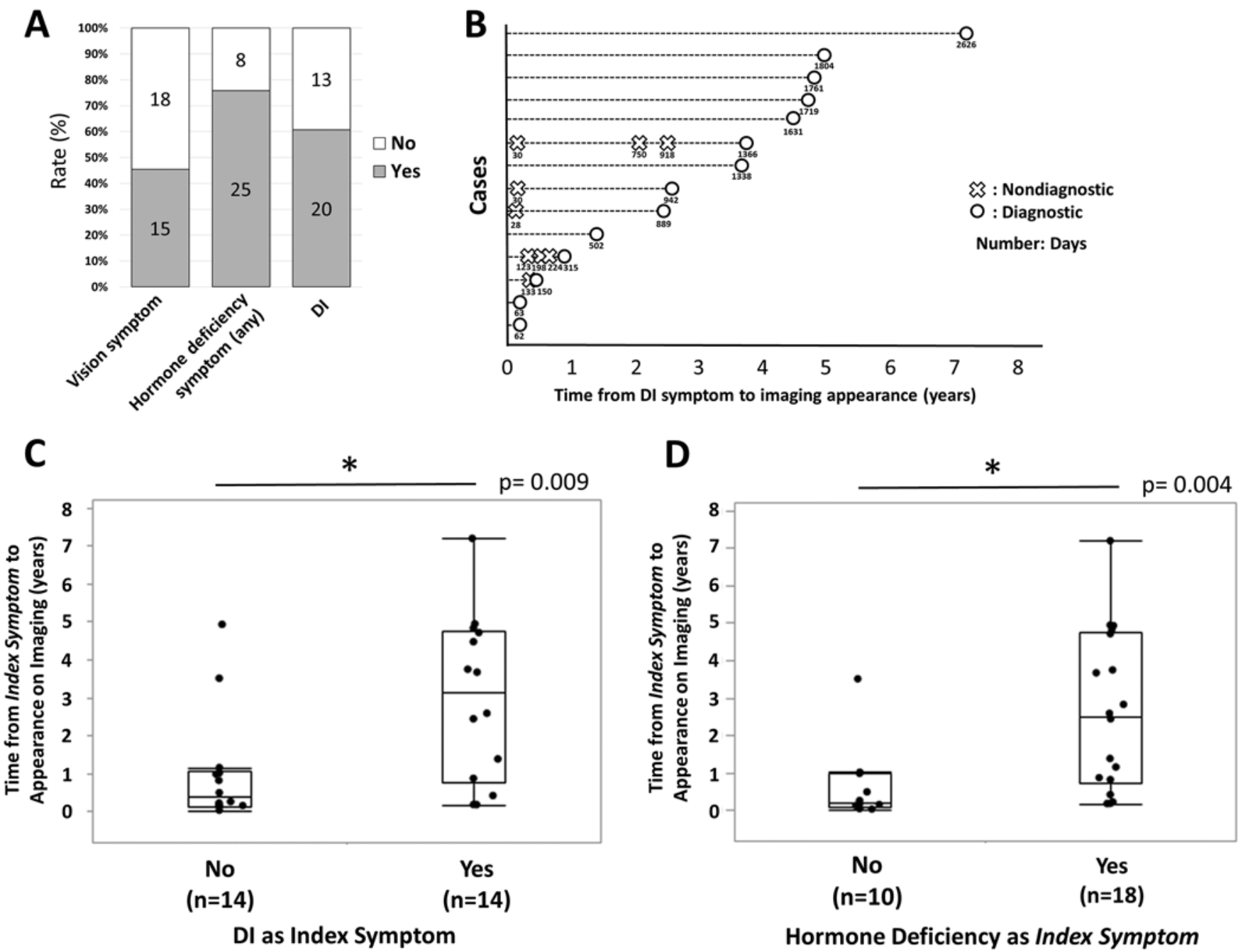

FIG. 2. A: Presenting symptoms, those documented at the time of primary medical examination, included visual deficits in 15 of 33 $(45 \%)$, hormone deficiency syndromes in 25 of $33(76 \%)$, and DI in 20 of $33(61 \%)$ patients. B: Individual patient plots (index cases) highlight the frequency of nondiagnostic imaging in radiographically latent DI-associated iGCT. C and D: Index symptoms, defined as the first disease feature noticed by the patient and reported in the patient's history, were reported in 28 patients, of whom 14 reported isolated $\mathrm{DI}(\mathrm{C}, 50 \%), 10$ identified isolated hormone deficiency $(\mathrm{D}, 36 \%)$, and 4 described concomitant $\mathrm{DI}$ and hormone deficiency symptoms ( $C$ and D, 14\%). Both DI and hormone deficiency as an index symptom were significantly associated with radiographic latency (360 vs 1083 days [p $=0.009$ ] and 245 vs 953 days $[p=0.004]$, respectively). ${ }^{*} p<0.05$.

\section{Discussion}

We present a single-institution retrospective case series of neurohypophyseal iGCTs, with a focus on the epidemiology, natural history, and prognostic factors. Our analysis demonstrates that, compared with other major iGCT subgroups, neurohypophyseal tumors predominantly occur in children, adolescents, and young adults, and, although a male sex predominance was noted in both groups, the distribution was more markedly skewed among patients with pineal iGCTs, greater than $90 \%$ of which arose in men, compared with approximately $75 \%$ of patients with neurohypophyseal iGCTs. Germinomas accounted for the vast majority of neurohypophyseal iGCTs $-89 \%$ overall, including all multifocal lesions-highlighting a potentially defining feature of neurohypophyseal lesions. This possibility is further reinforced when considered in con- trast to pineal region iGCTs, for which previous reports have described the germinoma incidence as $30 \%-40 \%$, which is in agreement with our institution's observation of 15 germinomas among 40 tumors (37.5\%) (Table 1). ${ }^{11,13,16}$

Although a significant proportion of patients described presenting symptoms that included visual deficit $(45 \%)$ or endocrinopathy (76\%), one of the most intriguing findings of our study was the prevalence of DI as an index symptom (50\%) and the association between early DI and prolonged radiographic latency. Sethi et al. reported that as much as $93 \%$ of patients with suprasellar iGCTs had polyuria and/or polydipsia symptoms, and $82 \%$ of patients with suprasellar iGCTs experienced delay in diagnosis $\geq$ 6 months..$^{25}$ On focused review, these challenging cases were frequently misdiagnosed as histiocytosis X or neurohypophysitis, given the propensity of the patients to present with imaging-negative pituitary dysfunction. In our 
A

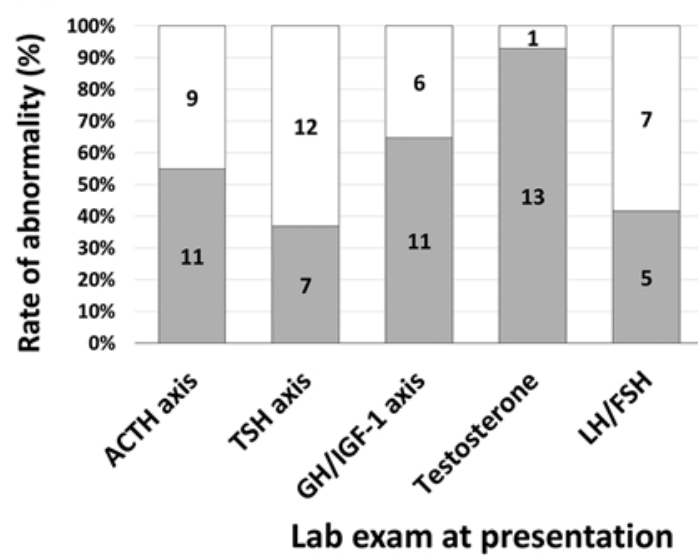

$\square$ Normal $\square$ Abnormal
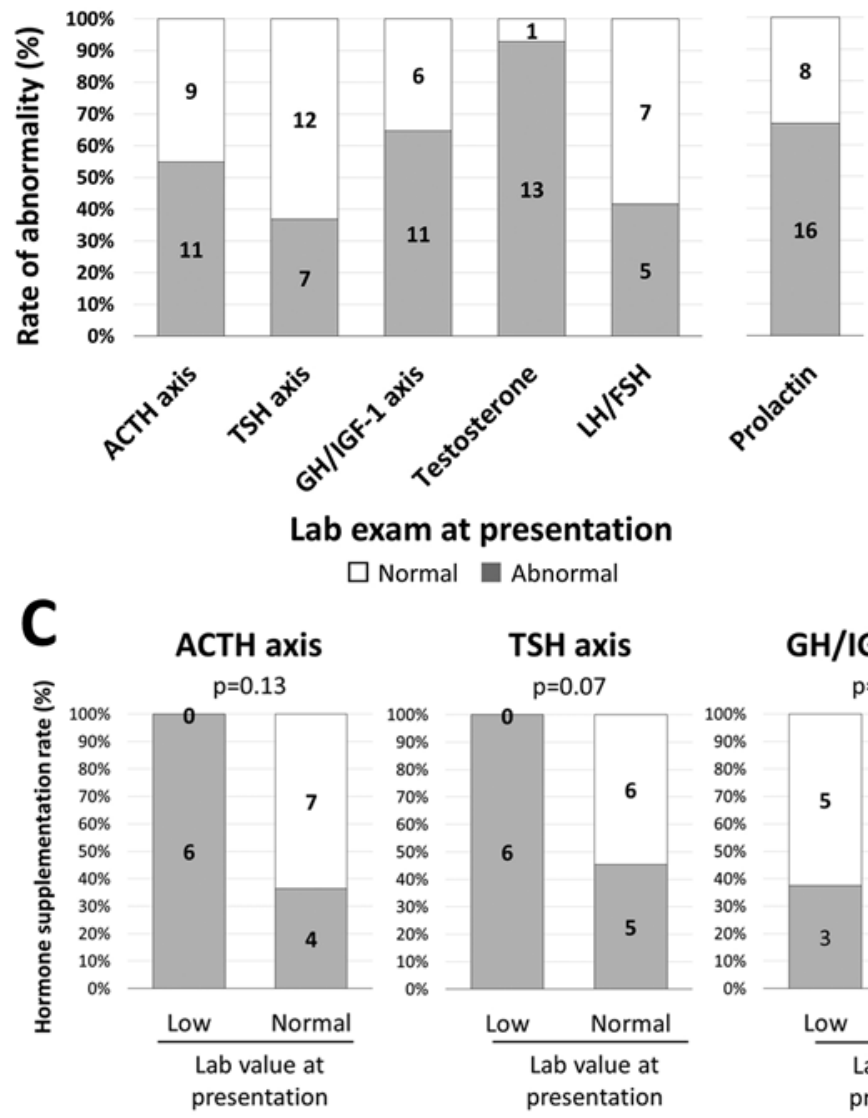

TSH axis

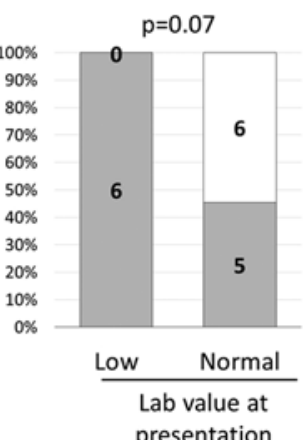

presentation
B

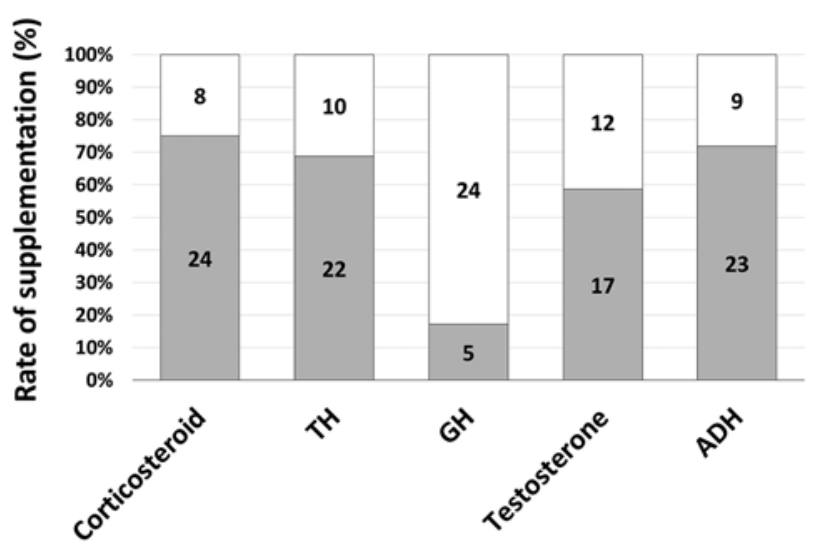

Type of hormone

$\square$ No $\square$ Yes

FIG. 3. Split bar graphs. A and B: The proportions of patients with biochemical evidence of pituitary dysfunction prior to treatment (A) or chronic posttreatment hormone supplementation (B). C: Correlation studies demonstrated a trend toward increased risk of posttreatment supplementation in association with pretreatment biochemical abnormalities; these differences did not reach statistical significance.

A

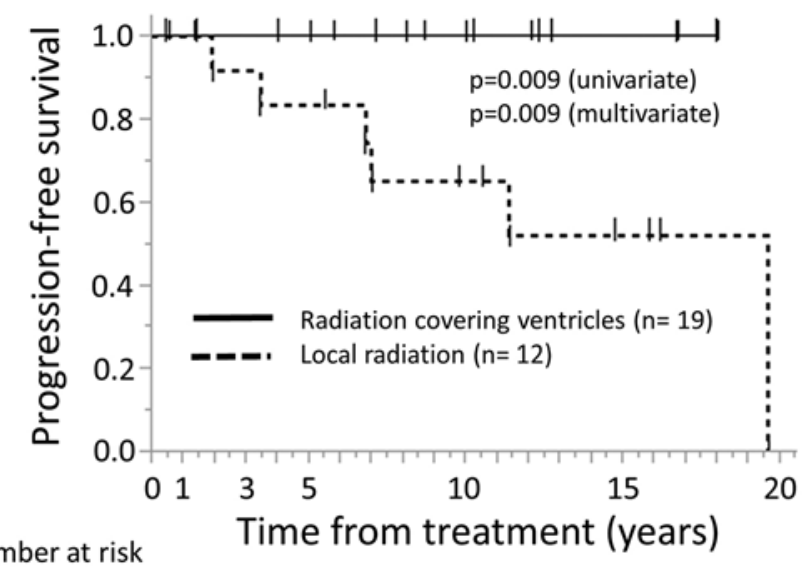

Number at risk

\begin{abstract}
$\begin{array}{llll}\text { WVI } & 19 & 19 & 15 \\ \end{array}$
\end{abstract}
Local radiation $\quad 1212 \quad 11 \quad 10$
GH/IGF-1 axis

$\mathrm{p}=0.22$

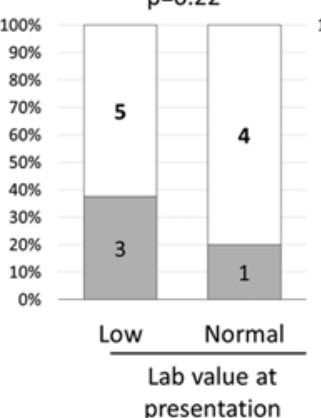

Testosterone

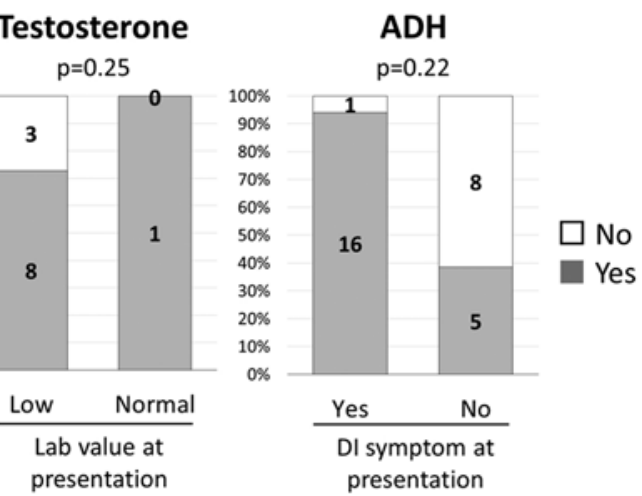


most extreme case, a patient underwent 3 MRI sessions during a latency period of 918 days from initial onset of DI until a lesion had finally grown large enough to be detected on the fourth study, nearly 4 years after the initial symptom. In part, this is attributable to technological limitations and the capacity for microscopic lesions to manifest dramatic hormone symptoms, particularly when compared with the significant mass effect required for iGCTs to produce visual deficits. ${ }^{14}$ Although rare, the natural history of prolonged latency prior to radiographic diagnosis is among the previously described trajectories for iGCTs, which Jennings et al. identified as particularly pronounced among suprasellar lesions, relative to other common iGCT locations. ${ }^{13}$ Yet another case report described a pineal iGCT associated with DI as an index symptom, which the authors speculated was likely attributable to an occult neurohypophyseal lesion. ${ }^{27}$ Indeed, even many confirmed iGCTs manifest little more radiographic evidence than a thickened infundibulum or the disappearance of posterior pituitary hyperintensity on T1-weighted sequences. ${ }^{10,17}$

With these findings in mind, we recommend close monitoring of pituitary function and serial imaging studies whenever iGCT is suspected, to both improve the probability of an early diagnosis and potentially avert the morbidity associated with extended pituitary dysfunction. ${ }^{17}$ Indeed, the relationship between DI and diagnosis is the core narrative describing how our understanding of iGCTs and their optimal management has evolved over the 3 decades encompassed within the study period of this analysis. More specifically, we have become uncompromisingly vigilant in our approach to serial imaging studies for DI with imaging studies negative for iGCT, particularly among patients 12-20 years of age. Furthermore, standardization of surveillance and treatment protocols via the COG has come to define standard of care, with marked benefits for patients and the shared knowledge base regarding this rare entity alike.

While most aspects of the COG protocols are pertinent to treatment features such as timing, sequence, and modality, a parallel area of evolution in our practice has been within the specific niche of patients requiring operative neurosurgical treatment. Defining patients requiring surgery is a function of diagnosis, which is generally reached via the stepwise progression of assessing for serum and CSF tumor markers, followed by consideration for tissue biopsy in equivocal cases. If initial laboratory studies confirm germinoma, our standard protocol is to proceed directly to radiotherapy; however, this is infrequently the case, but as most nonteratomatous iGCTs are radiosensitive, our preferred initial operation is a biopsy. Approach options include a transsylvian one for predominantly parasellar lesions versus an endoscopic transventricular biopsy in patients with a defined intraventricular component, as well as hydrocephalus. The endoscopic option, although suboptimal for certain anatomical configurations, has the benefits of being more minimally invasive while allowing for simultaneous performance of an endoscopic third ventriculostomy for treatment of hydrocephalus. Following tissue diagnosis, radiosensitive iGCTs go on to receive 6 cycles of COG protocol chemotherapy, followed by radiotherapy.

Mature teratomas are the predominant neurohypophy- seal iGCTs for which patients are returned to the operating for an attempt at definitive resection relatively early in their clinical trajectory. For most lesions, a transsylvian trajectory is the safest and most reliable means of protecting the optic nerves, optic chiasm, major blood vessels, and pituitary infundibulum. Patients with pronounced hydrocephalus or large intraventricular lesions may be candidates for an interhemispheric transventricular approach; however, visualization of the chiasm is challenging in this setting, and it should be reserved for carefully selected individuals with ideal tumor anatomy.

Turning to the implications of our findings in the anterior gland, we identified significant dysfunction in at least 1 pituitary axis among more than half of all patients at presentation, in particular, ACTH, GH/IGF-1, testosterone axis, and prolactin. Although our sample size was too small to reach statistical significance, we observed a trend in which preoperative biochemical abnormalities appeared to be more prevalent among patients who went on to require chronic hormone supplementation at 2 years posttreatment, predominantly for the replacement of corticosteroids, thyroid hormone, testosterone, or ADH. This is a retrospective, single-institution study, and is therefore subject to selection, recall, and other pertinent sources of bias; our findings require further exploration in a larger cohort. Despite this, we do emphasize the need to improve screening and surveillance of potential patients to better improve diagnostic accuracy, identify candidates for prompt treatment, and ensure close follow-up of those individuals at highest risk of postoperative endocrine failure. Other authors have reached similar conclusions, including Jorsal and Rørth, who identified one or more endocrine abnormalities in $>90 \%$ of cases at diagnosis (including DI, the most common), and Sawamura et al., who described chronic, supplementation-dependent endocrinopathy in 58 of 85 cases $(68 \%) .{ }^{14,24}$ Although these studies were not restricted to neurohypophyseal lesions, they provide supporting evidence for the need to include a complete endocrinological assessment at the time of diagnosis in all iGCTs, with a low threshold to initiate supplementation, and close posttreatment follow-up, ideally by a dedicated neuroendocrine team whenever possible.

The finding of our study that local radiation therapy is insufficient treatment for neurohypophyseal germinomas, both in terms of recurrence and survival, corroborates the ongoing clinical study in COG and SIOP (European Society for Paediatric Oncology), where localized germinoma is treated with WVI and boost to the primary site (ACNS1123). It is noteworthy that all observed recurrences occurred outside of the radiation field in patients who underwent local radiation therapy, whereas no cases with radiation therapy covering the ventricles recurred. Our study is the first to report this finding in the neurohypophyseal iGCT population, which echoes earlier work on field-associated survival differences reported in both the general iGCT population and intracranial germinomas in any location. ${ }^{4,2}, 2$ Still, other reports have demonstrated that, in germinomas treated with local radiotherapy, recurrences occur not just outside of the treatment fields ${ }^{6}$ but most frequently in the periventricular regions, supporting WVI as perhaps the most balanced strategy between the 
demands of tumor control and reducing radiation burden. ${ }^{3}$ With our results and these corroborating findings from the literature in mind, we strongly recommend against reduction of the radiation field in germinoma treatment protocols, given the mounting evidence that both PFS and OS benefit from ventricular coverage.

\section{Conclusions}

We report a 35-patient series of neurohypophyseal iGCTs, characterizing several unique features of these challenging and nuanced tumors. Our key findings include the propensity for radiographic latency of iGCT on presentation, particularly in patients who develop DI or pituitary insufficiency as their index symptom, the potential association between preoperative biochemical abnormalities and long-term dependence on hormone supplementation, and the survival advantages of WBI/WVI over local radiation in the specific subpopulation of germinomas. The major limitation of our study is sample size, an intrinsic challenge of studying a rare disease; additionally, the analysis was carried out retrospectively. Indeed, further study in larger cohorts is clearly required to expand, confirm, and better characterize these findings; notwithstanding, our analysis represents a small but critical step forward in our understanding of the natural history and best management practices in these rare tumors, which we anticipate will helpfully inform clinical decision-making and direct future iGCT research.

\section{References}

1. Acharya S, DeWees T, Shinohara ET, Perkins SM: Long-term outcomes and late effects for childhood and young adulthood intracranial germinomas. Neuro Oncol 17:741-746, 2015

2. Aizer AA, Sethi RV, Hedley-Whyte ET, Ebb D, Tarbell NJ, Yock TI, et al: Bifocal intracranial tumors of nongerminomatous germ cell etiology: diagnostic and therapeutic implications. Neuro Oncol 15:955-960, 2013

3. Alapetite C, Brisse H, Patte C, Raquin MA, Gaboriaud G, Carrie C, et al: Pattern of relapse and outcome of non-metastatic germinoma patients treated with chemotherapy and limited field radiation: the SFOP experience. Neuro Oncol 12:1318-1325, 2010

4. Balmaceda C, Heller G, Rosenblum M, Diez B, Villablanca JG, Kellie S, et al: Chemotherapy without irradiation-a novel approach for newly diagnosed CNS germ cell tumors: results of an international cooperative trial. The First International Central Nervous System Germ Cell Tumor Study. J Clin Oncol 14:2908-2915, 1996

5. Calaminus G, Frappaz D, Kortmann RD, Krefeld B, Saran F, Pietsch T, et al: Outcome of patients with intracranial nongerminomatous germ cell tumors-lessons from the SIOPCNS-GCT-96 trial. Neuro Oncol 19:1661-1672, 2017

6. Calaminus G, Kortmann R, Worch J, Nicholson JC, Alapetite C, Garrè ML, et al: SIOP CNS GCT 96: final report of outcome of a prospective, multinational nonrandomized trial for children and adults with intracranial germinoma, comparing craniospinal irradiation alone with chemotherapy followed by focal primary site irradiation for patients with localized disease. Neuro Oncol 15:788-796, 2013

7. Eom KY, Kim IH, Park CI, Kim HJ, Kim JH, Kim K, et al: Upfront chemotherapy and involved-field radiotherapy results in more relapses than extended radiotherapy for intracranial germinomas: modification in radiotherapy volume might be needed. Int J Radiat Oncol Biol Phys 71:667-671, 2008
8. Fukushima S, Yamashita S, Kobayashi H, Takami H, Fukuoka K, Nakamura T, et al: Genome-wide methylation profiles in primary intracranial germ cell tumors indicate a primordial germ cell origin for germinomas. Acta Neuropathol 133:445-462, 2017

9. Goldman S, Bouffet E, Fisher PG, Allen JC, Robertson PL, Chuba PJ, et al: Phase II trial assessing the ability of neoadjuvant chemotherapy with or without second-look surgery to eliminate measurable disease for nongerminomatous germ cell tumors: A Children's Oncology Group Study. J Clin Oncol 33:2464-2471, 2015

10. Gottschling S, Graf N, Meyer S, Reinhard H, Krenn T, Rohrer T: Intracranial germinoma: a rare but important differential diagnosis in children with growth retardation. Acta Paediatr 95:302-305, 2006

11. Hankinson EV, Lyons CJ, Hukin J, Cochrane DD: Ophthalmological outcomes of patients treated for pineal region tumors. J Neurosurg Pediatr 17:558-563, 2016

12. Ichimura K, Fukushima S, Totoki Y, Matsushita Y, Otsuka A, Tomiyama A, et al: Recurrent neomorphic mutations of MTOR in central nervous system and testicular germ cell tumors may be targeted for therapy. Acta Neuropathol 131:889-901, 2016

13. Jennings MT, Gelman R, Hochberg F: Intracranial germcell tumors: natural history and pathogenesis. J Neurosurg 63:155-167, 1985

14. Jorsal T, Rørth M: Intracranial germ cell tumours. A review with special reference to endocrine manifestations. Acta Oncol 51:3-9, 2012

15. Louis D, Ohgaki H, Wiestler O, Cavenee W: WHO Classification of Tumours of the Central Nervous System, rev ed 4. Lyon: International Agency for Research on Cancer, 2016

16. Matsutani M, Sano K, Takakura K, Fujimaki T, Nakamura O, Funata N, et al: Primary intracranial germ cell tumors: a clinical analysis of 153 histologically verified cases. J Neurosurg 86:446-455, 1997

17. Mootha SL, Barkovich AJ, Grumbach MM, Edwards MS, Gitelman SE, Kaplan SL, et al: Idiopathic hypothalamic diabetes insipidus, pituitary stalk thickening, and the occult intracranial germinoma in children and adolescents. J Clin Endocrinol Metab 82:1362-1367, 1997

18. Murray MJ, Bartels U, Nishikawa R, Fangusaro J, Matsutani M, Nicholson JC: Consensus on the management of intracranial germ-cell tumours. Lancet Oncol 16:e470-e477, 2015

19. Oosterhuis JW, Looijenga LH: Testicular germ-cell tumours in a broader perspective. Nat Rev Cancer 5:210-222, 2005

20. Oosterhuis JW, Stoop H, Honecker F, Looijenga LH: Why human extragonadal germ cell tumours occur in the midline of the body: old concepts, new perspectives. Int J Androl 30:256-264, 2007

21. Ostrom QT, Gittleman H, Liao P, Vecchione-Koval T, Wolinsky Y, Kruchko C, et al: CBTRUS Statistical Report: primary brain and other central nervous system tumors diagnosed in the United States in 2010-2014. Neuro Oncol 19 (Suppl 5):v1-v88, 2017

22. Phuakpet K, Larouche V, Hawkins C, Huang A, Tabori U, Bartels UK, et al: Rare presentation of supratentorial primitive neuroectodermal tumors mimicking bifocal germ cell tumors: 2 case reports. J Pediatr Hematol Oncol 38: e67e70, 2016

23. Reddy AT, Wellons JC III, Allen JC, Fiveash JB, Abdullatif $\mathrm{H}$, Braune KW, et al: Refining the staging evaluation of pineal region germinoma using neuroendoscopy and the presence of preoperative diabetes insipidus. Neuro Oncol 6:127-133, 2004

24. Sawamura Y, Ikeda J, Shirato H, Tada M, Abe H: Germ cell tumours of the central nervous system: treatment consideration based on 111 cases and their long-term clinical outcomes. Eur J Cancer 34:104-110, 1998 
25. Sethi RV, Marino R, Niemierko A, Tarbell NJ, Yock TI, MacDonald SM: Delayed diagnosis in children with intracranial germ cell tumors. J Pediatr 163:1448-1453, 2013

26. Shen H, Shih J, Hollern DP, Wang L, Bowlby R, Tickoo SK, et al: Integrated molecular characterization of testicular germ cell tumors. Cell Reports 23:3392-3406, 2018

27. Tarng DC, Huang TP: Diabetes insipidus as an early sign of pineal tumor. Am J Nephrol 15:161-164, 1995

28. Teilum G: Classification of endodermal sinus tumour (mesoblatoma vitellinum) and so-called "embryonal carcinoma" of the ovary. Acta Pathol Microbiol Scand 64:407-429, 1965

29. Uematsu Y, Tsuura Y, Miyamoto K, Itakura T, Hayashi S, Komai N: The recurrence of primary intracranial germinomas. Special reference to germinoma with STGC (syncytiotrophoblastic giant cell). J Neurooncol 13:247-256, 1992

30. Weksberg DC, Shibamoto Y, Paulino AC: Bifocal intracranial germinoma: a retrospective analysis of treatment outcomes in 20 patients and review of the literature. Int J Radiat Oncol Biol Phys 82:1341-1351, 2012

\section{Disclosures}

The authors report no conflict of interest concerning the materials or methods used in this study or the findings specified in this paper.

\section{Author Contributions}

Conception and design: Daniels, Takami. Acquisition of data: Takami. Analysis and interpretation of data: Takami. Drafting the article: Takami. Critically revising the article: all authors. Reviewed submitted version of manuscript: all authors. Approved the final version of the manuscript on behalf of all authors: Daniels. Statistical analysis: Takami, Graffeo. Administrative/ technical/material support: Daniels, Graffeo, Perry. Study supervision: Daniels, Graffeo, Perry.

\section{Correspondence}

David J. Daniels: Mayo Clinic, Rochester, MN. daniels.david@ mayo.edu. 